\title{
Epidemiology of Aortic Aneurysm Repair in the United States from 1993 to 2003
}

\author{
JOHN A. COWAN, JR., JUSTIN B. DIMICK, PETER K. HENKE, \\ JOHN RECTENWALD, JAMES C. STANLEY, \\ AND GILBERT R. UPCHURCH, Jr.
}

University of Michigan Cardiovascular Center, Ann Arbor, Michigan, USA

\begin{abstract}
The epidemiology of abdominal aortic aneurysm (AAA) disease has been well described over the preceding 50 years. This disease primarily affects elderly males with smoking, hypertension, and a positive family history contributing to an increased risk of aneurysm formation. The aging population as well as increased screening in high-risk populations has led some to suggest that the incidence of AAAs is increasing. The National Inpatient Sample (1993-2003), a national representative database, was used in this study to determine trends in mortality following AAA repair in the United States. In addition, the impact of the introduction of less invasive endovascular AAA repair was assessed. Overall rates of treated unruptured and ruptured AAAs remained stable (unruptured 12 to 15/100,000; ruptured 1 to 3/100,000). In 2003, 42.7\% of unruptured and $8.8 \%$ of ruptured AAAs were repaired through an endovascular approach. Inhospital mortality following unruptured AAA repair continues to decline for open repair $(5.3 \%$ to $4.7 \%, P=0.007)$. Mortality after elective endovascular AAA repair also has statistically decreased $(2.1 \%$ to $1.0 \%, P=0.024)$ and remains lower than open repair. Mortality rates for ruptured AAAs following repair remain high (open: $46.5 \%$ to $40.7 \%, P=0.01$; endovascular: $40.0 \%$ to $35.3 \%, P=$ $\mathbf{0 . 8 2 3})$. These data suggest that the numbers of patients undergoing elective AAA repair have remained relatively stable despite the introduction of less invasive technology. A shift in the treatment paradigm is occurring with a higher percentage of patients subjected to elective endovascular AAA repair compared to open repair. This shift, at least in the short term, appears justified as the mortality in patients undergoing elective endovascular AAA repair is significantly reduced compared to patients undergoing open AAA repair.
\end{abstract}

KEYWORDS: abdominal aortic aneurysm; repair; endovascular

Address for correspondence: Gilbert R. Upchurch, Jr., M.D., Section of Vascular Surgery, 1500 E. Medical Center Dr., Taubman Health Center, Ann Arbor, MI 48109-0329. Voice: 734-936-5790; fax: 734-647-9867.

e-mail: riversu@umich.edu

Ann. N.Y. Acad. Sci. 1085: 1-10 (2006). (C) 2006 New York Academy of Sciences.

doi: 10.1196/annals.1383.030 


\section{INTRODUCTION}

Dramatic improvements have been made during the past 50 years in the overall diagnosis, management, and treatment of abdominal aortic aneurysms (AAAs). Early detection programs, improvements in risk factor control, and a better understanding of the progression to rupture have allowed physicians and surgeons to make better treatment decisions. ${ }^{1-4}$ Furthermore, recognizing the potential benefits of regionalizing these complex procedures to high volume centers and specialized surgeons may further improve treatment outcomes. ${ }^{5-7}$ The advent and dissemination of endovascular approaches to AAAs will likely expand the number of patients in the population undergoing AAA repair. These facts will be crucial in managing the increasing elderly population at risk for AAAs in the United States.

Unfortunately, the mortality for ruptured AAAs has improved little in recent decades. ${ }^{2,5}$ While some evidence suggests the rate of ruptured AAAs in the general population is decreasing, certain populations, including those of lower socioeconomic status and the uninsured, have significantly higher rates of rupture. ${ }^{5,8}$ Measures to provide minimum preventative services or screening to manage AAAs before rupture may be needed if outcomes are to continue to improve. The objective of this investigation was to provide a population-level analysis of AAAs treated in the United States from 1993 to 2003.

\section{METHODS}

All clinical data were obtained from the Nationwide Inpatient Sample (NIS) for the years 1993 to 2003 . The NIS is a $20 \%$ random, stratified sample of discharges from nonfederal hospitals in the United States. ${ }^{9}$ The database is maintained by the Agency for Healthcare Research and Quality (AHRQ) as part of the Healthcare Costs and Utilization Project (HCUP). The clinical sample was derived in a two-step process using International Classification of Diseases, Ninth Revision, (ICD-9) procedure and diagnostic codes. First, all patients underwent repair of an AAA either open (ICD-9, 38.34 aorta resection and anastomosis; 38.44 replacement of abdominal aorta; 38.64 excision of aorta; 39.52 other repair of aneurysm) or endovascular (ICD-9, 39.71 endovascular abdominal aorta repair). The endovascular code did not come into use until the year 2000. Second, diagnostic codes for both unruptured AAAs (ICD-9, 441.4 abdominal aneurysm without mention of rupture; 441.9 aortic aneurysm of unspecified site without mention of rupture) and ruptured AAAs (ICD-9, 441.3 abdominal aneurysm, ruptured; 441.5 aortic aneurysm of unspecified site, ruptured; 441.6 thoracoabdominal aneurysm, rupture) were used to ensure the sample included only treatment for AAAs rather than other conditions. Population estimates of AAA repair utilization were made using discharge sampling weights provided by the NIS and information provided by 
the United States Census Bureau. ${ }^{10}$ Basic demographics were reported and tested for changes over time. Mortality, length of stay (LOS), and total charges were also calculated. Total charges were adjusted to the year 2003 dollar values using the consumer price index (CPI) for inpatient hospital services. ${ }^{11}$

Statistical analyses were carried out using analysis of variance (ANOVA) for patient age, chi-square for gender, race, and mortality, and Mann-Whitney U test for LOS and charges. All analyses were performed using SPSS software package (SPSS version 12.0, SPSS Inc., Chicago, IL, USA). P $<0.05$ was considered significant for all analyses.

\section{RESULTS}

The overall sample yielded 94,825 cases of treated AAAs (80,183 unruptured, 14,642 ruptured) over the 11 years of study. The mean age of the population was 72.0 years and was higher for patients with ruptured AAAs (TABLE 1). Females comprised $20.8 \%$ of patients. Overall mortality, LOS, and total charges were significantly higher for patients presenting with rupture.

Marked differences in the demographics and overall outcomes between open and endovascular repair were observed (TABLES 2 and 3). Years 2000 to 2003 were used in the comparisons since endovascular repair was not separately coded prior to that time. Patients who underwent endovascular repair for unruptured AAAs were more likely to be male and older. Mortality and LOS were less for endovascular repair, while total hospital charges were more for

TABLE 1. Demographics and outcomes for patients who underwent AAA repair in the NIS from 1993 to 2003

\begin{tabular}{lcccr}
\hline & & \multicolumn{2}{c}{ Aneurysm type } & \\
\cline { 3 - 4 } & Total & Unruptured & Ruptured & \multirow{2}{*}{$P^{*}$} \\
\hline Number & 94,825 & 80,183 & 14,642 & - \\
Patient age in years $\pm S D$ & $72.0(8.1)$ & $71.8(8.0)$ & $73.1(8.7)$ & $<0.001$ \\
Female (\%) & $20.8 \%$ & $20.7 \%$ & $21.4 \%$ & 0.063 \\
Caucasian race (\%) & $92.2 \%$ & $92.5 \%$ & $90.8 \%$ & $<0.001$ \\
Comorbid conditions & & & & \\
COPD $\dagger$ & $23.7 \%$ & $23.9 \%$ & $22.8 \%$ & 0.003 \\
Diabetes mellitus & $8.2 \%$ & $8.6 \%$ & $6.1 \%$ & $<0.001$ \\
History of myocardial infarction & $9.5 \%$ & $10.4 \%$ & $4.8 \%$ & $<0.001$ \\
Inhospital mortality & $10.4 \%$ & $4.2 \%$ & $44.8 \%$ & $<0.001$ \\
Median LOS in days $\ddagger$ & $7(5$ to 11$)$ & $7(5$ to 10$)$ & $9(2$ to 17$)$ & $<0.001$ \\
Median hospital charges $\ddagger$ & $\$ 44,977$ & $\$ 43,085$ & $\$ 65,004$ & $<0.001$ \\
& $(\$ 31,463$ to & $(\$ 30,754$ to & $(\$ 38,596$ & \\
& $\$ 68,736)$ & $\$ 63,062)$ & $\$ 121,658)$ & \\
\hline
\end{tabular}

*ANOVA for patient age; chi-square for gender, race, and mortality; Mann-Whitney U test for LOS and charges.

$\dagger \mathrm{COPD}=$ Chronic obstructive pulmonary disease $; \ddagger=$ interquartile range. 
TABLE 2. Demographics of patients who underwent open versus endovascular repair for an unruptured AAA in the NIS from 2000 to 2003

\begin{tabular}{lccc}
\hline & \multicolumn{2}{c}{ Aneurysm repair type } & \\
\cline { 2 - 3 } & Open & Endovascular & $P^{*}$ \\
\hline Number & 22,672 & 9,392 & - \\
Patient age in years $\pm S D$ & $71.7(8.2)$ & $73.4(8.1)$ & $<0.001$ \\
Female (\%) & $22.7 \%$ & $15.9 \%$ & $<0.001$ \\
Caucasian race (\%) & $90.8 \%$ & $90.9 \%$ & 0.878 \\
Comorbid conditions & & & \\
COPD $\dagger$ & $25.6 \%$ & $22.2 \%$ & $<0.001$ \\
Diabetes mellitus & $9.8 \%$ & $12.6 \%$ & $<0.001$ \\
History of myocardial infarction & $10.8 \%$ & $15.3 \%$ & $<0.001$ \\
Inhospital mortality & $4.4 \%$ & $1.3 \%$ & $<0.001$ \\
Median LOS in days $\ddagger$ & $7(5$ to 10$)$ & $2(1$ to 4$)$ & $<0.001$ \\
Median hospital charges $\ddagger$ & $\$ 43,462$ & $\$ 51,877$ & $<0.001$ \\
& $(\$ 30,913$ to $\$ 66,185)$ & $(\$ 38,369$ to $\$ 72,146)$ & \\
\hline
\end{tabular}

*ANOVA for patient age; chi-square for gender, race, and mortality; Mann-Whitney U test for LOS and charges.

$\dagger \mathrm{COPD}=$ Chronic obstructive pulmonary disease; $\ddagger=$ interquartile range.

endovascular repair. Patients treated for ruptured AAAs were of similar age, gender, and race comparing open and endovascular approaches. Mortality was less in the endovascular cohort, although did not reach statistical significance.

TABLE 3. Demographics of patients who underwent open versus endovascular repair for a ruptured AAA in the NIS from 2000 to 2003

\begin{tabular}{lccc}
\hline & \multicolumn{2}{c}{ Aneurysm repair type } & \\
\cline { 2 - 3 } & Open & Endovascular & $P^{*}$ \\
\hline Number & 4,439 & 271 & - \\
Patient age in years $\pm S D$ & $73.1(9.0)$ & $73.6(9.0)$ & 0.334 \\
Female (\%) & $22.8 \%$ & $23.3 \%$ & 0.851 \\
Caucasian race (\%) & $88.8 \%$ & $86.0 \%$ & 0.218 \\
Comorbid conditions & & & \\
COPD $\dagger$ & $24.6 \%$ & $26.6 \%$ & 0.471 \\
Diabetes mellitus & $7.6 \%$ & $9.6 \%$ & 0.230 \\
History of myocardial infarction & $5.0 \%$ & $7.4 \%$ & 0.078 \\
Inhospital mortality & $42.7 \%$ & $36.7 \%$ & 0.052 \\
Median LOS in days $\ddagger$ & $9(2$ to 18$)$ & $8(2$ to 15$)$ & 0.205 \\
Median hospital charges $\ddagger$ & $\$ 69,611$ & $\$ 71,926$ & 0.555 \\
& $(\$ 41,535$ to $\$ 129,935)$ & $(\$ 45,266$ to $\$ 132,925)$ & \\
& &
\end{tabular}

*ANOVA for patient age; chi-square for gender, race, and mortality; Mann-Whitney U test for LOS and charges.

$\dagger \mathrm{COPD}=$ Chronic obstructive pulmonary disease $\ddagger=$ interquartile range. 


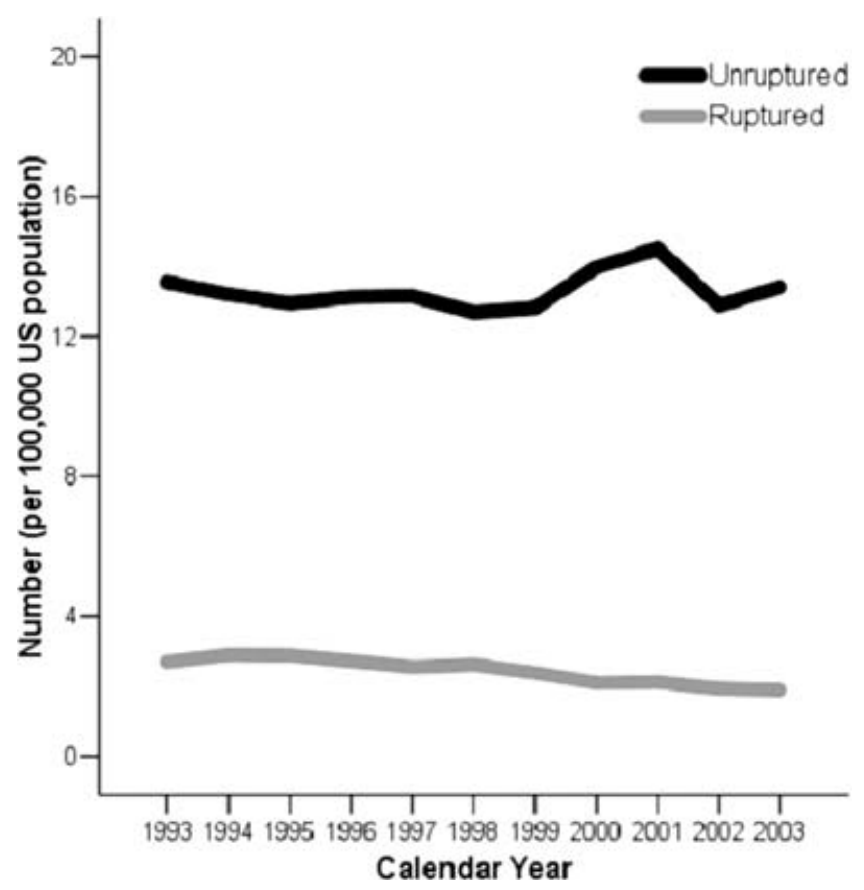

FIGURE 1. Estimated number of unruptured and ruptured AAAs treated in United States hospitals from 1993 to 2003.

Overall rates of treated unruptured and ruptured AAAs remained relatively constant during the period of study (unruptured 12 to 15/100,000; ruptured 1 to $3 / 100,000$ ) (FIG. 1). In 2003, 42.7\% of unruptured and $8.8 \%$ of ruptured AAAs were repaired using an endovascular approach (FIG. 2). Mortality for unruptured AAAs repaired by open means decreased from 5.3\% to $4.7 \%$ $(P=0.007)$. Endovascular AAA repair mortality rates from 2000 to 2003 were statistically decreased $(2.1 \%$ to $1.0 \%, P=0.024)$. Mortality rates for ruptured AAA treated by open repair decreased significantly, while the mortality for endovascular repair did not significantly change (open: $46.5 \%$ to $40.7 \%, P=$ 0.01 ; endovascular: $40.0 \%$ to $35.3 \%, P=0.823$ ) (FIG. 3 ).

\section{DISCUSSION}

AAA disease in the United States is still a disease primarily of the elderly male patient with more than 15,000 deaths in the year 2000 secondary to aortic disease. ${ }^{12}$ Other well-documented risk factors for the development of an AAA include tobacco use, the presence of hypertension, atherosclerosis in other vascular beds, and hypercholesterolemia. ${ }^{3}$ 
(A)

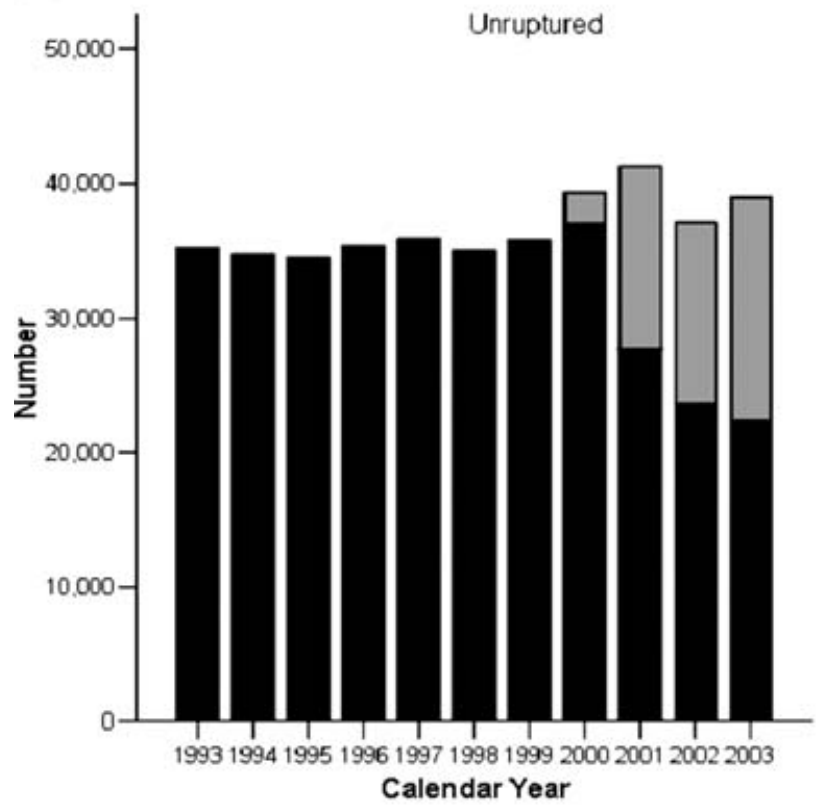

(B)

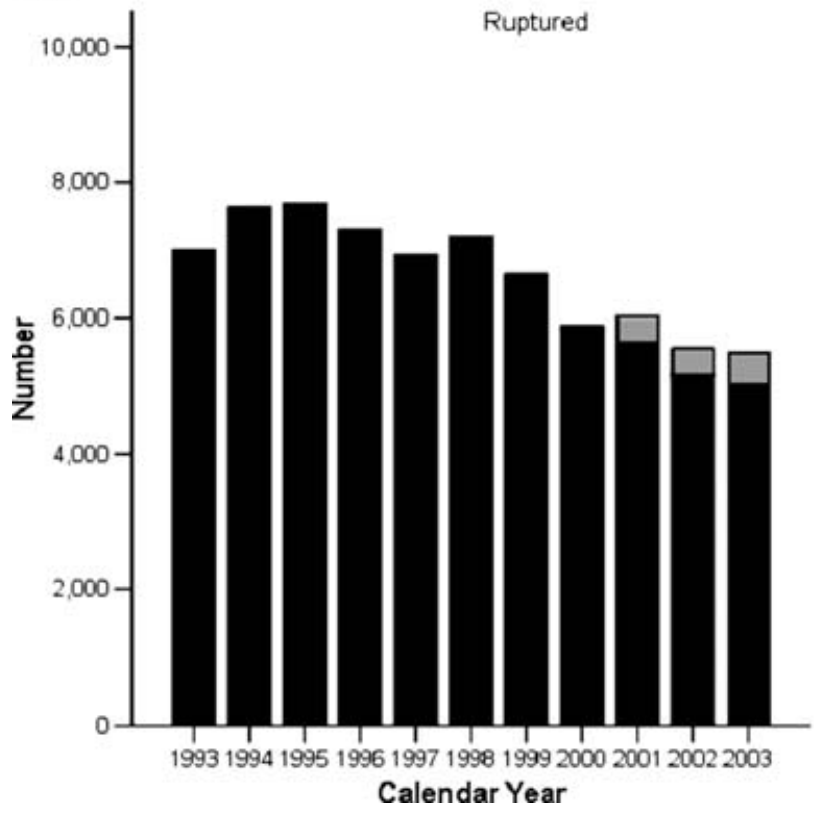

Open

Endo

Open

Endo

FIGURE 2. Estimated number of AAA repairs, by type, performed in the United States from 1993 to 2003. (A) Unruptured. (B) Ruptured. 


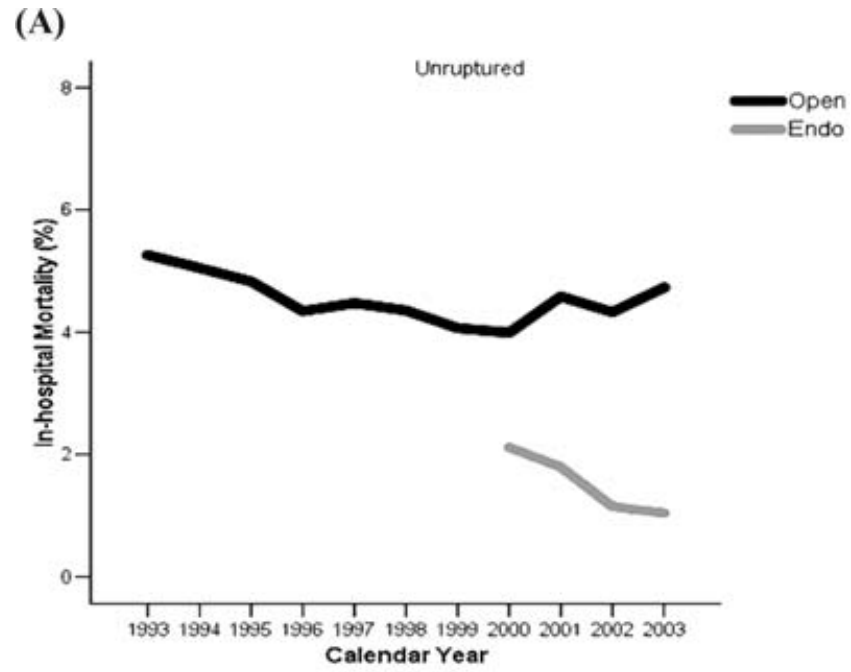

(B)

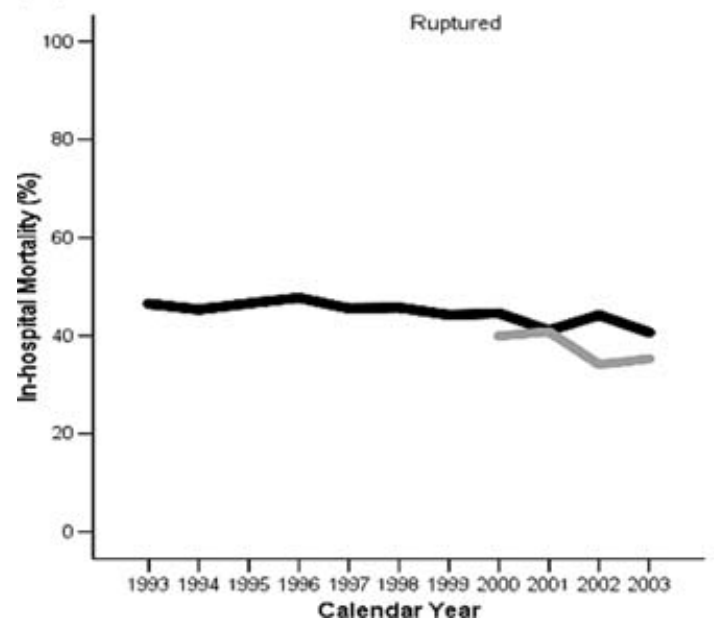

FIGURE 3. Inhospital mortality by repair type for (A) unruptured AAA (open: 5.3\% to $4.7 \%, P=0.007$; endovascular: $2.1 \%$ to $1.0 \%, P=0.024$ ). (B) ruptured AAA (open: $46.5 \%$ to $40.7 \%, P=0.01$; endovascular: $40.0 \%$ to $35.3 \%, P=0.823$ ).

Certain recent studies have suggested specific subsets of patients who may be at increased risk for AAA development and rupture. Englesbe reported on 1, 557 patients who underwent heart, kidney, or liver transplantation and were screened for AAAs. ${ }^{13}$ Cardiac transplant patients in particular, when screened for AAAs at a high rate ( $87 \%$ were screened), had a $5.8 \%$ prevalence of AAAs. In addition, among those transplant patients with AAAs, the rate of aneurysm rupture was $22.5 \%$ per year with a mean diameter of only $6.1 \mathrm{~cm}$. 
Uninsured patients may also be at greater risk for AAA rupture and dying more frequently even when their intact AAA is repaired electively. A recent study by Boxer and others suggested that patients without insurance or who had Medicaid compared to patients with private insurance presented more often with ruptured AAAs. ${ }^{8}$ Furthermore, patients without insurance had a higher mortality rate compared to patients with private insurance following elective AAA repair, even after correcting for comorbidities. These findings suggest screening programs designed to reduce the incidence of patients presenting with ruptured AAAs should perhaps target noninsured patients.

The incidence of AAAs in the general population of the United States should predictably be increasing with the aging population. However, the present data suggest that the numbers of patients undergoing elective AAA repair have remained relatively constant. This has been the case despite the introduction of less invasive diagnostic and therapeutic technology, which often serves to lower the threshold for intervention. Clearly, there are many examples in the vascular surgery literature in which a "less invasive" therapy, even if it is inferior in the long term to conventional operative therapy, has become the primary mode of treatment of a disease. Examples of the accelerated use of less invasive technology have been documented in the endovascular treatment of patients with aortoiliac occlusive disease ${ }^{14}$ as well as in the treatment of patients with renovascular hypertension secondary to renal artery stenosis. ${ }^{15}$ It is possible that the total number of patients undergoing AAA repair in the United States has recently remained stable despite the introduction of less invasive technology, secondary to a number of large randomized trials suggesting that the threshold diameter for AAA repair should be increased from $5 \mathrm{~cm}$ to $5.5 \mathrm{~cm}$, especially in males. ${ }^{4,16,17}$

A shift in the treatment paradigm of patients with AAAs is occurring over time with a higher percentage of patients undergoing elective endovascular AAA repair compared to open AAA repair. In the short term, this is clearly justified as mortality following elective endovascular AAA repair is significantly less than open AAA repair. This difference in outcome has occurred despite increased comorbidities such as prior myocardial infarction and more diabetics in the endovascular group. However, endovascular therapy entails greater costs reflected by increased hospital charges as documented in this study.

Clearly, the paradigm for elective AAA repair has changed. ${ }^{18-20}$ Nowygrod and colleagues examined national data from the NIS and statewide data from four states and also documented a significant shift in the treatment paradigm in patients undergoing elective AAA repair. ${ }^{18}$ While also using the NIS, but examining AAA repair only as the primary procedure code, the authors noted that endovascular AAA repair was used for $43 \%$ of total AAA repairs. Data from the Center for the Evaluative Clinical Sciences revealed that the rates of AAA repair per 1,000 Medicare enrollees, despite the introduction of endovascular AAA repair, were similar to levels observed in the mid 1990s. ${ }^{19}$ This report 
documented that inpatient reimbursements for AAA repair had fallen modestly since 1991. Dillavou and colleagues, also using the Medicare database, noted that endovascular AAA repair was more often undertaken in the elderly with lower mortality especially in older patients compared to open AAA repair. ${ }^{20}$ They also reported average elective AAA repair hospital charges that were not different for patients undergoing open or endovascular repair. However, endovascular repair was reimbursed more poorly by Medicare. This occurred even with more patients in the endovascular AAA group classified as DRG 111 (major cardiovascular procedure without complications), supporting a higher reimbursement level.

Importantly, two reasons may exist to explain why fewer patients are presenting with ruptured AAAs. First, strategies aimed at reducing modifiable risk factors such as quitting smoking and treating hypertension may have had a positive effect on aneurysm development and subsequent rupture. Second, screening programs may be having their intended affect, namely allowing early elective AAA repair and lessening the number of late ruptured AAAs. In the setting of relatively constant numbers of patients undergoing elective AAA repair, it appears that endovascular technology has not accelerated the rate of total AAA repair.

While not specifically addressed with the data from the current studies, individual centers of excellence have demonstrated remarkably low mortality rates following ruptured endovascular AAA repair. ${ }^{21,22}$ Although greater numbers of patients having ruptured AAAs will need to be treated endovascularly before significant decreases in mortality can be realized with the endovascular approach, this technology has the potential to significantly lower the staggering mortality accompanying open ruptured AAA repair.

\section{REFERENCES}

1. Brewster, D.C., J.L. Cronenwett, J.W. Hallett, JR., et al. 2003. Guidelines for the treatment of abdominal aortic aneurysms. Report of a subcommittee of the Joint Council of the American Association for Vascular Surgery and Society for Vascular Surgery. J. Vasc. Surg. 37: 1106-1117.

2. Heller, J.A., A. Weinberg, R. Arons, et al. 2000. Two decades of abdominal aortic aneurysm repair: have we made any progress? J. Vasc. Surg. 32: 10911100 .

3. Lederle, F.A., G.R. Johnson, S.E. Wilson, et al. 2000. The aneurysm detection and management study screening program: validation cohort and final results. Arch. Intern. Med. 160: 1425-1430.

4. Lederle, F.A., S.E. Wilson, G.R. Johnson, et al. 2002. Immediate repair compared with surveillance of small abdominal aortic aneurysms. N. Engl. J. Med. 346: $1437-1444$.

5. Wainess, R.M., J.B. Dimick, J.A. CowAn JR., et al. 2004. Epidemiology of surgically treated abdominal aortic aneurysms in the United States, 1988 to 2000. Vascular 12: 218-224. 
6. Dimick, J.B., J.A. Cowan, JR., J.C. Stanley, et al. 2003. Surgeon specialty and provider volumes are related to outcome of intact abdominal aortic aneurysm repair in the United States. J. Vasc. Surg. 38: 739-744.

7. BirKMEYER, J.D. \& J.B. Dimick. Potential benefits of the new Leapfrog standards: effect of process and outcomes measures. Surgery 135: 569-575.

8. BoXer, L.K., J.B. Dimick, R.M. WAiness, et al. 2003. Payer status is related to differences in access and outcomes of abdominal aortic aneurysm repair in the United States. Surgery 134: 142-145.

9. Healthcare Cost, and Utilization Project (HCUP). Nationwide Inpatient Sample, 1993-2003. Rockville, MD: Agency for Health Care Research and Quality.

10. U.S. Census Bureau. Population estimates. Available at: http://eire.census. gov/popest/estimates.php (accessed July 1, 2004).

11. U.S. Department of Labor. Bureau of Labor Statistics: http://www.bls.gov (accessed February 2004).

12. National Center for Health Statistics. Deaths, percent of total deaths, and death rates for the 10 leading causes of death in selected age groups, by race and sex: United States. 2000. National Vital Statistics Report, 2002. Accessed at www.cdc.gov/nchs/fastats/pdf/nvsr50'16t1.pdf on 15 November 2004.

13. Englesbe, M.J., A.H. Wu, A.W. Clowes \& R.E. Zierler. 2003. The prevalence and natural history of aortic aneurysms in heart and abdominal organ transplant patients. J. Vasc. Surg. 37: 27-31.

14. Upchurch, G.R., JR., J.B. Dimick, R.M. WAiness, et al. 2004. Diffusion of new technology in health care: the case of aorto-iliac occlusive disease. Surgery 136: 812-818.

15. KnipP, B.S., J.B. Dimick, J.L. Eliason, et al. 2004. Diffusion of new technology for the treatment of renovascular hypertension in the United States: surgical revascularization versus catheter-based therapy, 1988-2001. J. Vasc. Surg. 40: $717-723$.

16. Ashton, H.A. , M.J. Buxton, N.E. Day, et al. 2002. The Multicentre Aneurysm Screening Study (MASS) into the effect of abdominal aortic aneurysm screening on mortality in men: a randomised controlled trial. Lancet 360: 1531-1539.

17. United Kingdom Small Aneurysm Trial Participants. 2002. Long-term outcomes of immediate repair compared with surveillance of small abdominal aortic aneurysms. N. Engl. J. Med. 346: 1445-1452.

18. Nowygrod, R., N. Egorova, G. Greco, et al. 2006. Trends, complications, and mortality in peripheral vascular surgery. J. Vasc. Surg. 43: 205-216.

19. Center for the Evaluative Clinical Sciences. Dartmouth- CMS- FDA Collaborative. 2005. Trends and Regional Variations in Abdominal Aortic Aneurysm Repair. Dartmouth Atlas of Health Care: Studies of Surgical Variation. 1-24.

20. Dillavou, E.D., S.C. Muluk \& M.S. Makaroun. 2006. Improving aneurysmrelated outcomes: nationwide benefits of endovascular repair. J. Vasc. Surg. 43: 446-452.

21. Ohкi, T., F.J. Veith, L.A. SAnCheZ, et al. 1999. Endovascular graft repair of ruptured aortoiliac aneurysms. J. Am. Coll. Surg. 189: 102-113.

22. Hechelhammer, L., M.L. Lachat, S. Wildermuth, et al. 2005. Midterm outcome of endovascular repair of ruptured abdominal aortic aneurysms. J. Vasc. Surg. 41: 752-757. 